\title{
Correction to: AGGF1 protects from myocardial ischemia/reperfusion injury by regulating myocardial apoptosis and angiogenesis
}

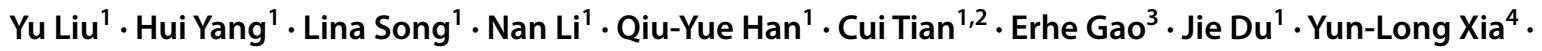 \\ Hui-Hua Li ${ }^{1,2}$
}

Accepted: 26 October 2021 / Published online: 6 November 2021

(c) Springer Science+Business Media, LLC, part of Springer Nature 2021

\section{Correction to: Apoptosis (2014) 19:1254-1268 https://doi.org/10.1007/s10495-014-1001-4}

The original version of this article unfortunately contains errors in figures.

The images of TUNEL, $a-a c t i n i n$, and DAPI staining in both sham and isotype $+\mathrm{I} / \mathrm{R}$ in Fig. $3 \mathrm{~A}$, and the images of immunohistochemical staining for ischemic hearts with anti-Mac-2 antibody in 3.0-treated groups after I/
R+anti-AGGGF1 (mg/kg) in Fig. 6b were shown incorrectly. The correct Fig. 3A and Fig. 6B are given below. The corrections do not affect either the validity of the data or the overall conclusions.
The original article can be found online at https://doi.org/10.1007/ s10495-014-1001-4.

Yun-Long Xia

yunlong_xia@126.com

$\triangle$ Hui-Hua Li

hhli1995@yahoo.com

1 Department of Pathology, Physiology and Pathophysiology, Beijing, AnZhen Hospital the Key Laboratory of Remodeling-Related Cardiovascular Diseases, School of Basic Medical Sciences, Capital Medical University, No.10 Xitoutiao, You An Men, Beijing 100069, China

2 Department of Cardiology, Chaoyang Hospital, Capital Medical University, No.10 Xitoutiao, You An Men, Beijing 100069, China

3 Center for Translational Medicine, Temple University School of Medicine, Philadelphia, PA 19140, USA

4 Department of Cardiology, Institute of Cardiovascular Diseases, First Affiliated Hospital of Dalian Medical University, Dalian 116011, China 
Fig. 3 AGGF1 attenuates cardiomyocyte apoptosis in the heart after I/R injury. A Representative photomicrographs of cardiomyocyte apoptosis (top) in the ischemic area of hearts from sham, control, antiAGGF1 and rhAGGF1 examined by TUNEL assay (green). Myocytes were identified by immunostaining with anti-a-actinin antibody (red), and nuclei were counterstained with DAPI (blue). Quantitative analysis of TUNEL-positive cells (bottom). Bar 50 lm. Data are expressed as mean \pm SEM (n $=4$ per group). ${ }^{*} P<0.05$ versus sham mice; ${ }^{\#} P<0.05$ versus control mice.
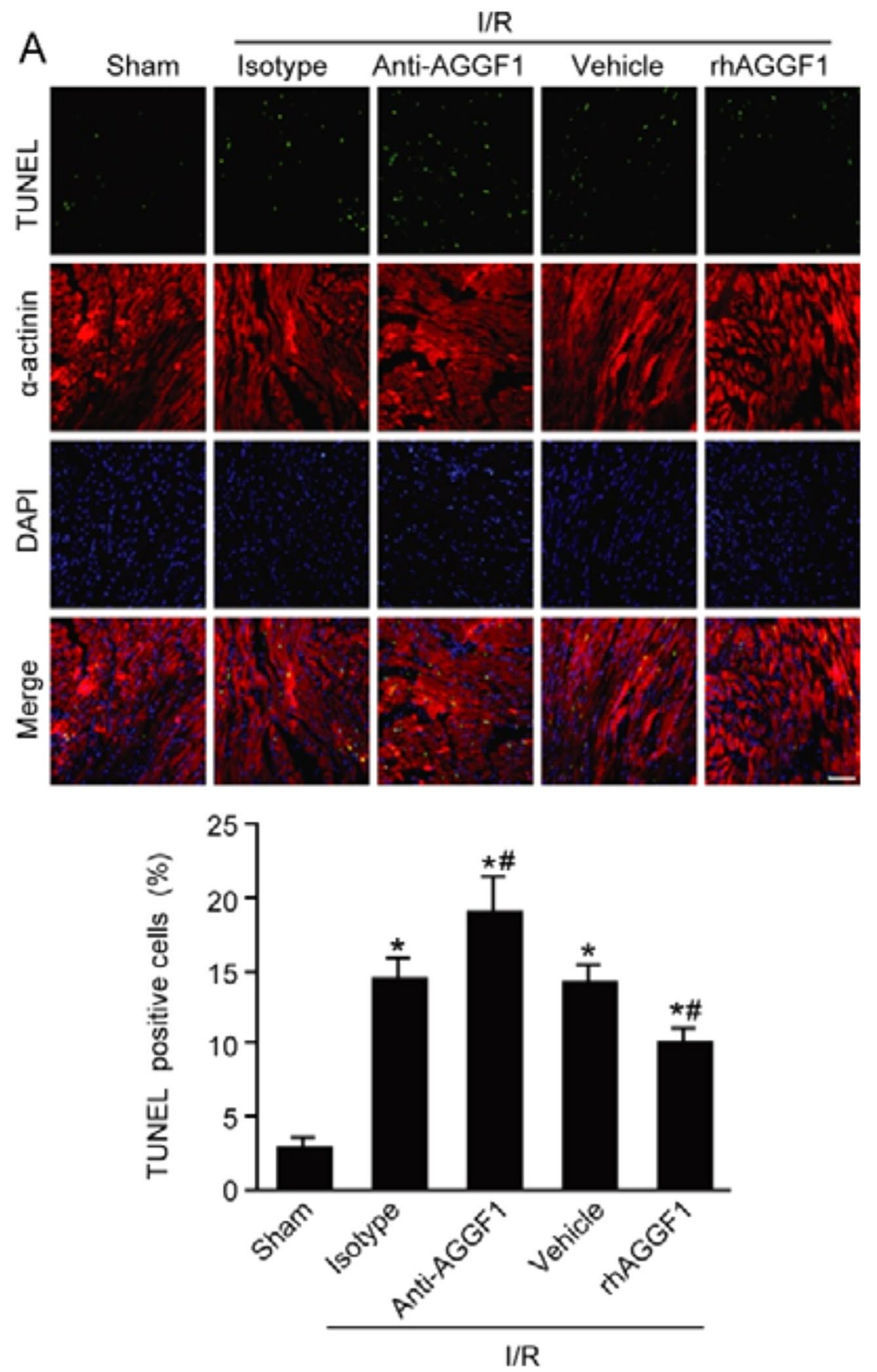

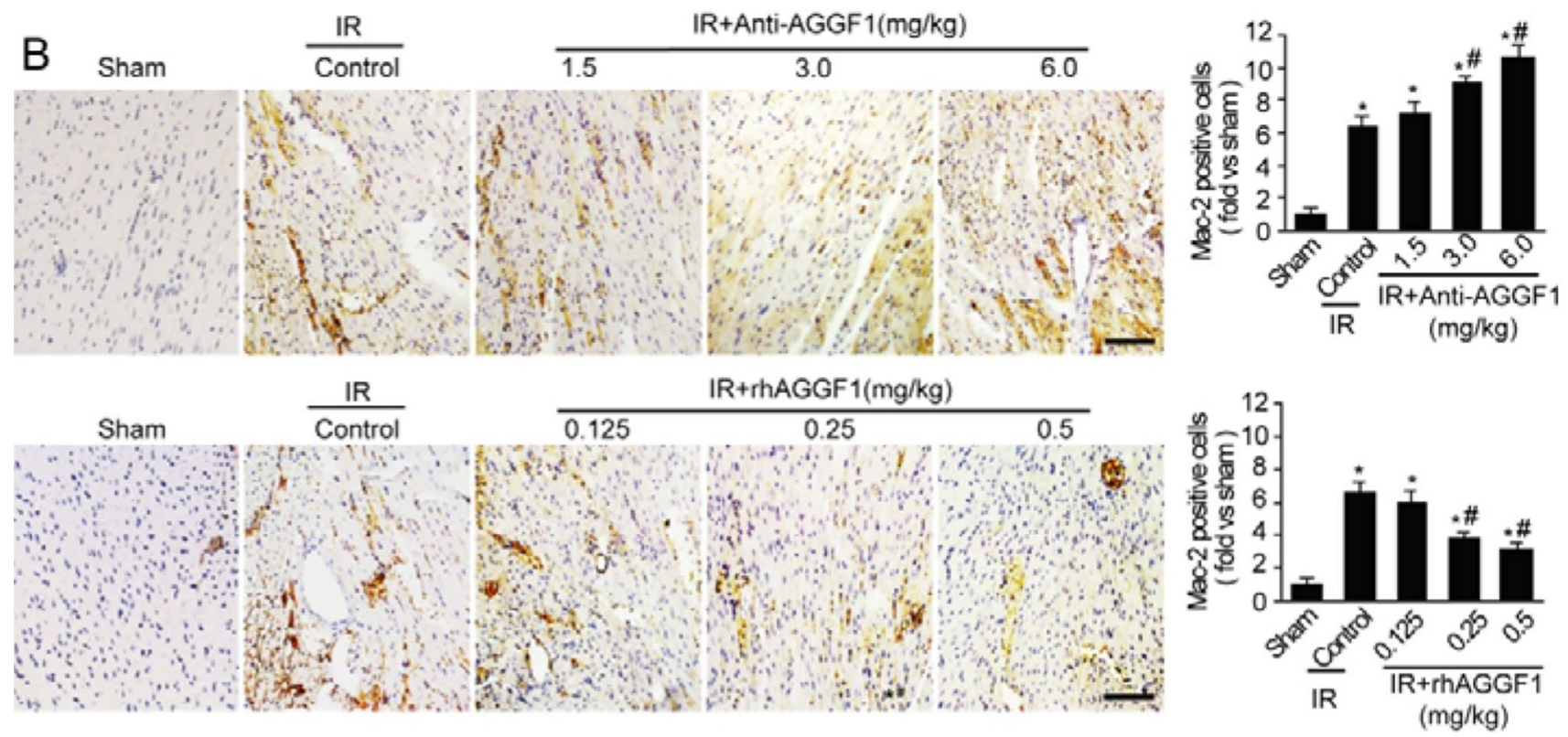

Fig. 6 AGGF1 attenuates cardiomyocyte apoptosis or inflammation and enhances angiogenesis after I/R injury. B Immunohistochemical staining of ischemic hearts from all groups with anti-Mac-2 antibody (left). Quantification of Mac-2-positive cells (right). Bars $50 \mu \mathrm{m}$. Data are expressed as mean \pm SEM $\left(\mathrm{n}=4\right.$ per group). ${ }^{*} P<0.05$ versus sham mice; ${ }^{\#} P<0.05$ versus control mice.

Publisher's Note Springer Nature remains neutral with regard to jurisdictional claims in published maps and institutional affiliations. 